\title{
Significant Advances in Medical Image Analysisorem
}

\author{
Dr. Stefan Bordag \\ ExB Research \& Development GmbH, Leipzig \\ bordag@exb.de
}

In the past 4 years Deep Learning (DL) has re-entered the computer vision scene dramatically, by completely shifting the design paradigm compared to the last 20 years. Whereas before the error rates in image analysis were more or less stagnant, since 2012 DL kept halving them each year, in some recent cases even achieving super-human performance! All typical tasks such classification, detection and segmentation benefited across all related applications such as traffic sign recognition, natural image analysis, automatic captioning. These developments move computer vision from a scientific playground to a productizable technology.

The two key winning factors of DL are the complete removing of feature engineering and the stacking of multiple layers of abstraction. The previous approaches relied entirely on the ability of the scientist in designing the most appropriate features to capture characteristic textures, edges, colours, etc. DL-based approaches obsolete the feature engineering process by learning any necessary features automatically. Through their hierarchical organisation the networks also learn to automatically differentiate abstraction levels.

From this follows, that this approach can be generalized to any other imagebased analysis problem. We are a high-tech company developing Artificial Intelligence solutions for the analysis of the visual and natural language data in the medical domain. We decided to investigate the largely unexplored medical domain with respect to DL. We demonstrate the disruptive new developments for medical image analysis on two exemplary tasks, mitosis detection and gland segmentation, where we also successfully participated in an image segmentation competition at MICCAI 2015. We show how deep learning dramatically improves the quality of results while also significantly reducing development efforts and time to market. Finally we discuss error analysis and outline further developments. 